\title{
Taste non-Goldstone pion decay constants in staggered chiral perturbation theory
}

\author{
Jon A. Bailey, Boram Yoon*, Weonjong Lee \\ Lattice Gauge Theory Research Center, CTP, and FPRD, \\ Department of Physics and Astronomy, Seoul National University, Seoul, 151-747, South Korea \\ E-mail: boramsnuegmail.com
}

\section{SWME Collaboration}

We calculate the next-to-leading order axial current decay constants of taste non-Goldstone pions and kaons in staggered chiral perturbation theory. This is an extension of the taste Goldstone decay constants calculation to that of the non-Goldstone tastes. We present results for the partially quenched case in the SU(3) and SU(2) staggered chiral perturbation theories and discuss the difference between the taste Goldstone and non-Goldstone cases.

The 30th International Symposium on Lattice Field Theory - Lattice 2012

June 24 - 29, 2012

Cairns, Australia

${ }^{*}$ Speaker. 


\section{Introduction}

Staggered chiral perturbation theory (SChPT) was developed to describe lattice data generated by staggered fermions, which have an exact chiral symmetry at nonzero lattice spacing. Using SChPT, lattice results can be extrapolated to the physical quark masses and the continuum limit, removing dominant lattice artifacts coming from the taste symmetry breaking of staggered fermions.

In Ref. [1], Aubin and Bernard calculated next-to-leading order (NLO) corrections to the decay constants of taste Goldstone pions and kaons, associated with the exact chiral symmetry of the staggered action, in SChPT. Here we extend the calculation to the taste non-Goldstone pions and kaons.

In Sec. 2, we consider the leading order (LO) and NLO terms of the chiral Lagrangian that contribute to the decay constants. In Sec. 3, we outline the calculation of NLO corrections to the decay constants of taste non-Goldstone pions and kaons, and write results in a theory with three flavors and four tastes for each flavor. In Sec. 4, we present the results for the partially quenched case in the SU(3) and SU(2) SChPT, and we conclude in Sec. 5. Unless defined explicitly we use the notation of Ref. [2].

\section{Chiral Lagrangian for staggered quarks}

The chiral Lagrangian for staggered quarks was formulated by Lee and Sharpe for the singleflavor case [3] and generalized by Aubin and Bernard to multiple flavors [4]. In the standard power counting,

$$
\mathscr{O}\left(p^{2} / \Lambda_{\chi}^{2}\right) \approx \mathscr{O}\left(m_{q} / \Lambda_{\chi}\right) \approx \mathscr{O}\left(a^{2} \Lambda_{\chi}^{2}\right)
$$

the order of a Lagrangian operator is the sum of non-negative integers, $n_{p^{2}}, n_{m}$ and $n_{a^{2}}$, which are the number of derivative pairs, number of quark mass factors, and powers of the squared lattice spacing in the operator, respectively. At LO, the Lagrangian operators fall into three classes: $\left(n_{p^{2}}, n_{m}, n_{a^{2}}\right)=(1,0,0),(0,1,0)$ and $(0,0,1)$, and we have

$$
\mathscr{L}_{\mathrm{LO}}=\frac{f^{2}}{8} \operatorname{Tr}\left(\partial_{\mu} \Sigma \partial_{\mu} \Sigma^{\dagger}\right)-\frac{1}{4} \mu f^{2} \operatorname{Tr}\left(M \Sigma+M \Sigma^{\dagger}\right)+\frac{2 m_{0}^{2}}{3}\left(U_{I}+D_{I}+S_{I}\right)^{2}+a^{2}\left(\mathscr{U}+\mathscr{U}^{\prime}\right),
$$

where $f$ is the decay constant at LO, $\mu$ is a constant in the unit of mass, $M$ is the mass matrix, $\Sigma \equiv \exp (i \phi / f)$, and $\phi$ is the pseudo-Goldstone boson (PGB) field. The term multiplied by $m_{0}^{2}$ is the anomaly contribution, and $\mathscr{U}$ and $\mathscr{U}^{\prime}$ are the taste symmetry breaking potentials defined in Ref. [4].

At NLO, there are six classes that satisfy $n_{p^{2}}+n_{m}+n_{a^{2}}=2$. Operators in two classes contribute to the decay constants: $\left(n_{p^{2}}, n_{m}, n_{a^{2}}\right)=(1,1,0)$ and $(1,0,1)$. The contributing operators in the class $(1,1,0)$ are Gasser-Leutwyler terms [5],

$$
\mathscr{L}_{\mathrm{GL}}=L_{4} \operatorname{Tr}\left(\partial_{\mu} \Sigma^{\dagger} \partial_{\mu} \Sigma\right) \operatorname{Tr}\left(\chi^{\dagger} \Sigma+\chi \Sigma^{\dagger}\right)+L_{5} \operatorname{Tr}\left(\partial_{\mu} \Sigma^{\dagger} \partial_{\mu} \Sigma\left(\chi^{\dagger} \Sigma+\Sigma^{\dagger} \chi\right)\right),
$$

where $L_{4}$ and $L_{5}$ are low-energy constants (LECs) and $\chi=2 \mu M$. The contributing operators in the class $(1,0,1)$ are terms given by Sharpe and Van de Water in Ref. [6]. 


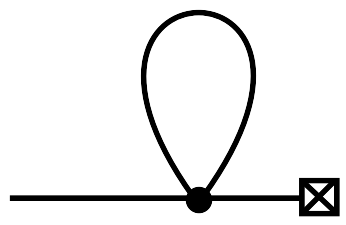

(a)

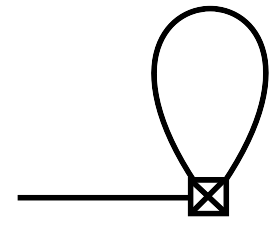

(b)

Figure 1: Diagrams contributing to the decay constants. (a) is the wavefunction renormalization correction and (b) is the current correction.

\section{Decay constants of flavor-charged pseudo-Goldstone bosons}

The decay constant $f_{P_{t}^{+}}$for a flavor-charged PGB $P_{t}^{+}$with taste $t$ is defined by the matrix elements

$$
\left\langle 0\left|j_{\mu 5, t}^{P^{+}}\right| P_{t}^{+}(p)\right\rangle=-i f_{P_{t}^{+}} p_{\mu},
$$

where $j_{\mu 5, t}^{P^{+}}$is the axial current. From the LO Lagrangian, the LO axial current is

$$
j_{\mu 5, t}^{P^{+}}=-i \frac{f^{2}}{8} \operatorname{Tr}\left[T^{t(3)} \mathscr{P}^{P^{+}}\left(\partial_{\mu} \Sigma \Sigma^{\dagger}+\Sigma^{\dagger} \partial_{\mu} \Sigma\right)\right]
$$

where $T^{a(3)} \equiv I_{3} \otimes T^{a}, I_{3}$ is the identity matrix in flavor space, and $\mathscr{P} P^{+}$is a projection operator that chooses $P^{+}$from the $\Sigma$ field, defined by $\mathscr{P}_{i j}^{P^{+}}=\delta_{i x} \delta_{j y}$. For flavor-charged states, which are the interest in this paper, $x \neq y$. Note that $\Sigma=\exp (i \phi / f)$ can be expanded in terms of $\phi$.

There are three types of NLO corrections to the decay constants: (a) one-loop wavefunction renormalization correction from the $\mathscr{O}(\phi)$ term of the axial current, $\delta f_{P_{t}^{+}}^{Z}$, (b) one-loop correction from the $\mathscr{O}\left(\phi^{3}\right)$ term of the axial current, $\delta f_{P_{t}^{+}}^{\text {current }}$, and (c) analytic contribution from the NLO terms of the axial current and the analytic contribution to the self-energy, $\delta f_{P_{t}^{+}}^{\text {anal }}$. Combining these three types of corrections (a) - (c), we write the decay constants:

$$
f_{P_{t}^{+}}=f\left[1+\frac{1}{16 \pi^{2} f^{2}}\left(\delta f_{P_{t}^{+}}^{Z}+\delta f_{P_{t}^{+}}^{\text {current }}\right)+\delta f_{P_{t}^{+}}^{\text {anal }}\right] .
$$

First we consider the wavefunction renormalization correction. Considering the $\mathscr{O}(\phi)$ term of the LO axial current, $j_{\mu 5, t}^{P^{+}, \phi}=f\left(\partial_{\mu} \phi_{y x}^{t}\right)$, we find contributions to the decay constants,

$$
\left\langle 0\left|j_{\mu 5, t}^{P^{+}, \phi}\right| P_{t}^{+}(p)\right\rangle=f\left(-i p_{\mu}\right)\left\langle 0\left|\phi_{y x}^{t}\right| P_{t}^{+}(p)\right\rangle=f\left(-i p_{\mu}\right) \sqrt{Z_{P_{t}^{+}}} .
$$

Here $Z_{P_{t}^{+}} \equiv 1+\delta Z_{P_{t}^{+}}$is the wavefunction renormalization constant of the $\phi$ field. $\delta Z_{P_{t}^{+}}$gives the NLO correction to the decay constants,

$$
\delta f_{P_{t}^{+}}^{Z} \equiv \frac{16 \pi^{2} f^{2}}{2} \delta Z_{P_{t}^{+}}=-\frac{16 \pi^{2} f^{2}}{2} \frac{d \Sigma\left(p^{2}\right)}{d p^{2}}
$$

where $\Sigma\left(p^{2}\right)$ is the self-energy of $P_{t}^{+}$. Using the self-energy from Ref. [2], we find the one-loop corrections

$$
\delta f_{P_{t}^{+}}^{Z}=\frac{1}{24} \sum_{a}\left[\sum_{Q} l\left(Q_{a}\right)+16 \pi^{2} \int \frac{d^{4} q}{(2 \pi)^{4}}\left(D_{x x}^{a}+D_{y y}^{a}-2 \theta^{a t} D_{x y}^{a}\right)\right]
$$


where $l\left(Q_{a}\right)$ is the chiral logarithm and $D_{i j}^{a}$ is the disconnected piece of the propagator. Here $Q$ runs over six flavor combinations, $x i$ and $y i$ for $i \in\{u, d, s\}, a$ runs over the 16 PGB tastes in the 15 and 1 of $S U(4)_{T}$, and $Q_{a}$ is the squared tree-level meson mass with flavor $Q$ and taste $a$. The self-energy also contains NLO analytic corrections to the decay constants, which we discuss below.

Next we consider the loop correction from the $\mathscr{O}\left(\phi^{3}\right)$ terms of the LO axial current,

$$
j_{\mu 5, t}^{P^{+}, \phi^{3}}=-\frac{1}{24 f} \tau_{t a b c}\left(\partial_{\mu} \phi_{y k}^{a} \phi_{k l}^{b} \phi_{l x}^{c}-2 \phi_{y k}^{a} \partial_{\mu} \phi_{k l}^{b} \phi_{l x}^{c}+\phi_{y k}^{a} \phi_{k l}^{b} \partial_{\mu} \phi_{l x}^{c}\right) .
$$

The contractions in the calculation of the matrix element defined in Eq. (3.1) give the loop integrals. Performing the loop integrals, we find the NLO current correction to the decay constants,

$$
\delta f_{P_{t}^{+}}^{\text {current }} \equiv-\frac{1}{6} \sum_{a}\left[\sum_{Q} l\left(Q_{a}\right)+16 \pi^{2} \int \frac{d^{4} q}{(2 \pi)^{4}}\left(D_{x x}^{a}+D_{y y}^{a}-2 \theta^{a t} D_{x y}^{a}\right)\right] .
$$

Note that $\delta f_{P_{t}^{+}}^{\text {current }}$ is proportional to $\delta f_{P_{t}^{+}}^{Z}$, which was shown for the taste Goldstone case in Ref. [1].

Now we consider the NLO analytic contributions to the decay constants. The terms from the NLO Lagrangian noted in Sec. 2 (including the $\mathscr{O}\left(p^{2} a^{2}\right)$ source operators) give the analytic contributions. The contributions of $\mathscr{O}\left(p^{2} a^{2}\right)$ terms may be written as $f a^{2} \mathscr{F}_{t}$. Here $\mathscr{F}_{t}$ are linear combinations of the LECs of the Lagrangian, which are degenerate within the irreps of the lattice symmetry group. As commented in Ref. [6], there are no relations between the $\mathrm{SO}(4)$-violations in the pion masses and the $\mathrm{SO}(4)$-violations in the axial current decay constants, due to the contributions from the $\mathscr{O}\left(p^{2} a^{2}\right)$ source operators.

The terms in Gasser-Leutwyler Lagrangian given in Eq. (2.3) contribute to the decay constants through wavefunction renormalization and the current. The wavefunction renormalization correction of the Gasser-Leutwyler terms can be calculated from the self-energy [2]. Collecting all the NLO analytic corrections to the decay constants, we find

$$
\delta f_{P_{t}^{+}}^{\text {anal }}=\frac{64}{f^{2}} L_{4} \mu\left(m_{u}+m_{d}+m_{s}\right)+\frac{8}{f^{2}} L_{5} \mu\left(m_{x}+m_{y}\right)+a^{2} \mathscr{F}_{t} .
$$

\section{Results}

The results given in Eqs. (3.6), (3.8) and (3.9) are the results in the 4+4+4 theory. In order to formulate the results in the $1+1+1$ theory (rooted staggered chiral perturbation theory), we use the replica method [7, 8, 9]. Applying the replica method to Eqs. (3.6), (3.8) and (3.9), we find

$$
\begin{aligned}
\delta f_{P_{F}^{+}} & =\delta f_{P_{F}^{+}}^{\text {con }}+\delta f_{P_{F}^{+}}^{\text {disc }}, \\
\delta f_{P_{t}^{+}}^{\text {anal }} & =\frac{16}{f^{2}} L_{4} \mu\left(m_{u}+m_{d}+m_{s}\right)+\frac{8}{f^{2}} L_{5} \mu\left(m_{x}+m_{y}\right)+a^{2} \mathscr{F}_{t} .
\end{aligned}
$$

where

$$
\begin{aligned}
& \delta f_{P_{F}^{+}}^{\mathrm{con}} \equiv-\frac{1}{32} \sum_{Q, B} g_{B} l\left(Q_{B}\right), \\
& \delta f_{P_{F}^{+}}^{\mathrm{disc}} \equiv-2 \pi^{2} \int \frac{d^{4} q}{(2 \pi)^{4}}\left(D_{x x}^{I}+D_{y y}^{I}-2 D_{x y}^{I}\right. \\
&\left.+4 D_{x x}^{V}+4 D_{y y}^{V}-2 \Theta^{V F} D_{x y}^{V}+4 D_{x x}^{A}+4 D_{y y}^{A}-2 \Theta^{A F} D_{x y}^{A}\right) .
\end{aligned}
$$


Table 1: The coefficient $\Theta^{B F}$ defined in Eq. (4.6) is in row $B$ and column $F$.

\begin{tabular}{c|rrrrr}
\hline \hline$B \backslash F$ & $V$ & $A$ & $T$ & $P$ & $I$ \\
\hline$V$ & -2 & 2 & 0 & -4 & 4 \\
$A$ & 2 & -2 & 0 & -4 & 4 \\
$T$ & 0 & 0 & -2 & 6 & 6 \\
$P$ & -1 & -1 & 1 & 1 & 1 \\
$I$ & 1 & 1 & 1 & 1 & 1 \\
\hline \hline
\end{tabular}

Here we performed the summation over $a$ within each taste $\mathrm{SO}(4)$ irrep for Eqs. (3.6) and (3.8), $B$ and $F$ represent the taste $S O(4)_{T}$ irreps,

$$
B, F \in\{I, V, T, A, P\},
$$

$t \in F$ and

$$
\Theta^{B F} \equiv \sum_{a \in B} \theta^{a t}, \quad g_{B} \equiv \sum_{a \in B} 1
$$

The coefficients $\Theta^{B F}$ are given in Table 1. The superscripts con and disc in $\delta f_{P_{F}^{+}}^{\text {con }}$ and $\delta f_{P_{F}^{+}}^{\text {disc }}$ represent connected and disconnected quark-flow contributions, respectively [1].

First, we consider partially quenched results for $1+1+1$ and $2+1$ flavor cases in SU(3) SChPT. The connected contributions to the decay constants in the partially quenched $1+1+1$ flavor case are the same as the Eq. (4.3). The disconnected contributions for the partially quenched $1+1+1$ flavor case are obtained by performing the integrals in Eq. (4.4) keeping all quark masses distinct,

$$
\begin{aligned}
\delta f_{P_{F}^{+}, m_{x} \neq m_{y}}^{\mathrm{disc}}=\sum_{Z}\left[\frac{1}{6}\left\{D_{X \pi^{0} \eta, X}^{U D S}\left(Z_{I}\right) l\left(Z_{I}\right)+D_{Y \pi^{0} \eta, Y}^{U D S}\left(Z_{I}\right) l\left(Z_{I}\right)-2 R_{X Y \pi^{0} \eta}^{U D S}\left(Z_{I}\right) l\left(Z_{I}\right)\right\}\right. \\
+\frac{1}{4} a^{2} \delta_{V}^{\prime}\left\{2 D_{X \pi^{0} \eta \eta^{\prime}, X}^{U D S}\left(Z_{V}\right) l\left(Z_{V}\right)+2 D_{Y \pi^{0} \eta \eta^{\prime}, Y}^{U D S}\left(Z_{V}\right) l\left(Z_{V}\right)-\Theta^{V F} R_{X Y \pi^{0} \eta \eta^{\prime}}^{U D S}\left(Z_{V}\right) l\left(Z_{V}\right)\right\} \\
+(V \rightarrow A)]+\frac{1}{6}\left\{R_{X \pi^{0} \eta}^{U D S}\left(X_{I}\right) \tilde{l}\left(X_{I}\right)+R_{Y \pi^{0} \eta}^{U D S}\left(Y_{I}\right) \tilde{l}\left(Y_{I}\right)\right\} \\
+\frac{1}{2} a^{2} \delta_{V}^{\prime}\left\{R_{X \pi^{0} \eta \eta^{\prime}}^{U D S}\left(X_{V}\right) \tilde{l}\left(X_{V}\right)+R_{Y \pi^{0} \eta \eta^{\prime}}^{U D S}\left(Y_{V}\right) \tilde{l}\left(Y_{V}\right)\right\}+(V \rightarrow A) .
\end{aligned}
$$

For $m_{x}=m_{y}$, we find

$$
\delta f_{P_{F}^{+}, m_{x}=m_{y}}^{\mathrm{disc}}=\frac{1}{4} a^{2} \delta_{V}^{\prime}\left(4-\Theta^{V F}\right)\left[R_{X \pi^{0} \eta \eta^{\prime}}^{U D S}\left(X_{V}\right) \tilde{l}\left(X_{V}\right)+\sum_{Z} D_{X \pi^{0} \eta \eta^{\prime}, X}^{U D S}\left(Z_{V}\right) l\left(Z_{V}\right)\right]+(V \rightarrow A) .
$$

For the $2+1$ flavor case, the connected contributions are obtained by setting $m_{u}=m_{d}$ in Eq. (4.3), and the disconnected contributions are obtained by setting $m_{u}=m_{d}$ and performing 
the integrals in Eq. (4.4). For $m_{x} \neq m_{y}$, we find

$$
\begin{aligned}
\delta f_{P_{F}^{+}, m_{x} \neq m_{y}}^{\mathrm{disc}}=\sum_{Z}\left[\frac{1}{6}\left\{D_{X \eta, X}^{\pi S}\left(Z_{I}\right) l\left(Z_{I}\right)+D_{Y \eta, Y}^{\pi S}\left(Z_{I}\right) l\left(Z_{I}\right)-2 R_{X Y \eta}^{\pi S}\left(Z_{I}\right) l\left(Z_{I}\right)\right\}\right. \\
+\frac{1}{4} a^{2} \delta_{V}^{\prime}\left\{2 D_{X \eta \eta^{\prime}, X}^{\pi S}\left(Z_{V}\right) l\left(Z_{V}\right)+2 D_{Y \eta \eta^{\prime}, Y}^{\pi S}\left(Z_{V}\right) l\left(Z_{V}\right)-\Theta^{V F} R_{X Y \eta \eta^{\prime}}^{\pi S}\left(Z_{V}\right) l\left(Z_{V}\right)\right\} \\
+(V \rightarrow A)]+\frac{1}{6}\left\{R_{X \eta}^{\pi S}\left(X_{I}\right) \tilde{l}\left(X_{I}\right)+R_{Y \eta}^{\pi S}\left(Y_{I}\right) \tilde{l}\left(Y_{I}\right)\right\} \\
+\frac{1}{2} a^{2} \delta_{V}^{\prime}\left\{R_{X \eta \eta^{\prime}}^{\pi S}\left(X_{V}\right) \tilde{l}\left(X_{V}\right)+R_{Y \eta \eta^{\prime}}^{\pi S}\left(Y_{V}\right) \tilde{l}\left(Y_{V}\right)\right\}+(V \rightarrow A) .
\end{aligned}
$$

For $m_{x}=m_{y}$, we find

$$
\delta f_{P_{F}^{+}, m_{x}=m_{y}}^{\mathrm{disc}}=\frac{1}{4} a^{2} \delta_{V}\left(4-\Theta^{V F}\right)\left[R_{X \eta \eta^{\prime}}^{\pi S}\left(X_{V}\right) \tilde{l}\left(X_{V}\right)+\sum_{Z} D_{X \eta \eta^{\prime}, X}^{\pi S}\left(Z_{V}\right) l\left(Z_{V}\right)\right]+(V \rightarrow A) .
$$

Next, we consider the partially quenched results for the $1+1+1$ flavor case in SU(2) SChPT. The connected contributions to the decay constants are obtained by dropping terms corresponding to strange sea quark loops from Eq. (4.3). The disconnected contributions are obtained from Eqs. (4.7), (4.8) and (4.4), by taking the $\mathrm{SU}(2)$ limit treating $x$ and $y$ as light quarks $\left(m_{x}, m_{y}, m_{u}, m_{d} \ll\right.$ $\left.m_{s}\right)$,

$$
\begin{aligned}
\delta f_{P_{F}^{+}, m_{x} \neq m_{y}}^{\mathrm{disc}}=\sum_{Z}\left[\frac{1}{4}\left\{D_{X \pi^{0}, X}^{U D}\left(Z_{I}\right) l\left(Z_{I}\right)+D_{Y \pi^{0}, Y}^{U D}\left(Z_{I}\right) l\left(Z_{I}\right)-2 R_{X Y \pi^{0}}^{U D}\left(Z_{I}\right) l\left(Z_{I}\right)\right\}\right. \\
\left.\quad+\frac{1}{4} a^{2} \delta_{V}^{\prime}\left\{2 D_{X \pi^{0} \eta, X}^{U D}\left(Z_{V}\right) l\left(Z_{V}\right)+2 D_{Y \pi^{0} \eta, Y}^{U D}\left(Z_{V}\right) l\left(Z_{V}\right)-\Theta^{V F} R_{X Y \pi^{0} \eta}^{U D}\left(Z_{V}\right) l\left(Z_{V}\right)\right\}+(V \rightarrow A)\right] \\
+\frac{1}{4}\left\{R_{X \pi^{0}}^{U D}\left(X_{I}\right) \tilde{l}\left(X_{I}\right)+R_{Y \pi^{0}}^{U D}\left(Y_{I}\right) \tilde{l}\left(Y_{I}\right)\right\} \\
+\frac{1}{2} a^{2} \delta_{V}^{\prime}\left\{R_{X \pi^{0} \eta}^{U D}\left(X_{V}\right) \tilde{l}\left(X_{V}\right)+R_{Y \pi^{0} \eta}^{U D}\left(Y_{V}\right) \tilde{l}\left(Y_{V}\right)\right\}+(V \rightarrow A),
\end{aligned}
$$

and

$$
\delta f_{P_{F}^{+}, m_{x}=m_{y}}^{\mathrm{disc}}=\frac{1}{4} a^{2} \delta_{V}^{\prime}\left(4-\Theta^{V F}\right)\left[R_{X \pi^{0} \eta}^{U D}\left(X_{V}\right) \tilde{l}\left(X_{V}\right)+\sum_{Z} D_{X \pi^{0} \eta, X}^{U D}\left(Z_{V}\right) l\left(Z_{V}\right)\right]+(V \rightarrow A) .
$$

For the $2+1$ flavor case in SU(2) SChPT, the connected contributions are obtained by setting $m_{u}=m_{d}$ for the $1+1+1$ case, and the disconnected contributions are obtained by setting $m_{u}=m_{d}$ in Eqs. (4.7) and (4.8),

$$
\begin{aligned}
\delta f_{P_{F}, m_{x} \neq m_{y}}^{\mathrm{disc}}=\sum_{Z}\left[-\frac{1}{2} R_{X Y}^{\pi}\left(Z_{I}\right) l\left(Z_{I}\right)\right. \\
\left.\quad+\frac{1}{4} a^{2} \delta_{V}^{\prime}\left\{2 D_{X \eta, X}^{\pi}\left(Z_{V}\right) l\left(Z_{V}\right)+2 D_{Y \eta, Y}^{\pi}\left(Z_{V}\right) l\left(Z_{V}\right)-\Theta^{V F} R_{X Y \eta}^{\pi}\left(Z_{V}\right) l\left(Z_{V}\right)\right\}+(V \rightarrow A)\right] \\
+\frac{1}{4}\left\{l\left(X_{I}\right)+\left(\pi_{I}-X_{I}\right) \tilde{l}\left(X_{I}\right)+l\left(Y_{I}\right)+\left(\pi_{I}-Y_{I}\right) \tilde{l}\left(Y_{I}\right)\right\} \\
+\frac{1}{2} a^{2} \delta_{V}^{\prime}\left\{R_{X \eta}^{\pi}\left(X_{V}\right) \tilde{l}\left(X_{V}\right)+R_{Y \eta}^{\pi}\left(Y_{V}\right) \tilde{l}\left(Y_{V}\right)\right\}+(V \rightarrow A)
\end{aligned}
$$


and

$$
\delta f_{P_{F}, m_{x}=m_{y}}^{\mathrm{disc}}=\frac{1}{4} a^{2} \delta_{V}^{\prime}\left(4-\Theta^{V F}\right)\left[R_{X \eta}^{\pi}\left(X_{V}\right) \tilde{l}\left(X_{V}\right)+\sum_{Z} D_{X \eta, X}^{\pi}\left(Z_{V}\right) l\left(Z_{V}\right)\right]+(V \rightarrow A) .
$$

\section{Conclusion}

In Eqs. (4.7) - (4.10), we present the NLO corrections to the pion and kaon decay constants for the partially quenched case calculated in the SU(3) SChPT; in Eqs. (4.11) - (4.14), we present the NLO corrections to the pion and kaon decay constants for the partially quenched case calculated in the SU(2) SChPT. As one can see in Eqs. (4.4) and (4.2), the only differences between taste Goldstone and taste non-Goldstone cases are the $\Theta^{B F}$ factors multiplying $D_{x y}^{A, V}$ and the generalized constants $\mathscr{F}_{t}$ in the analytic contribution. $\Theta^{B F}$ originates from the trace of taste generators and affects only the flavor-charged disconnected propagator, $D_{x y}^{A, V} . \mathscr{F}_{t}$ are degenerate within the lattice symmetry group, and there are no relations between the $\mathrm{SO}(4)$-violations in the pion masses and the $\mathrm{SO}(4)$-violations in the axial current decay constants. Using these results, it is possible to improve determinations of the decay constants, quark masses and the Gasser-Leutwyler constants by analyzing lattice data from taste non-Goldstone channels.

\section{Acknowledgments}

W. Lee is supported by the Creative Research Initiatives Program (2012-0000241) of the NRF grant funded by the Korean government (MEST), and acknowledges support from KISTI supercomputing center through the strategic support program for the supercomputing application research [No. KSC-2011-G2-06].

\section{References}

[1] C. Aubin and C. Bernard, Phys. Rev. D68, (2003), 074011 ; [hep-lat/0306026].

[2] J. Bailey, et al., SWME Collaboration, Phys. Rev. D85, (2012), 094503 ; [arXiv: 1112 . 2108 ].

[3] W. Lee and S. Sharpe, Phys. Rev. D60, (1999), 114503 ; [hep-lat/9905023].

[4] C. Aubin and C. Bernard, Phys. Rev. D68, (2003), 034014 ; [hep-lat/ 0304014 ].

[5] J. Gasser and H. Leutwyler, Nucl. Phys. B250, (1985), 465

[6] S. Sharpe and R. Van de Water, Phys. Rev. D71, (2005), 114505 ; [hep-lat/ 0409018 ].

[7] C. Bernard and M. Golterman, Phys. Rev. D49, (1994), 486-494 ; [hep-lat/93060 05].

[8] P. Damgaard and K. Splittorff, Phys. Rev. D62, (2000), 054509 ; [hep-lat/ 0003017$].$

[9] C. Bernard, M. Golterman and Y. Shamir, Phys. Rev. D77, (2008), 074505 ; [arXiv: 0712 . 2560 ]. 